\title{
A prospective study on the role of Hysterolaparoscopy in the evaluation of infertility
}

\author{
Pandeeswari B.*, Shalini Mahana Valecha
}

Department of Obstetrics and Gynecology, ESI-PGIMSR, Mumbai, Maharashtra, India

Received: 18 September 2019

Revised: 13 October 2019

Accepted: 06 November 2019

\author{
*Correspondence: \\ Dr. Pandeeswari B., \\ E-mail: pandeeswaribalakrishnan@gmail.com
}

Copyright: ( $)$ the author(s), publisher and licensee Medip Academy. This is an open-access article distributed under the terms of the Creative Commons Attribution Non-Commercial License, which permits unrestricted non-commercial use, distribution, and reproduction in any medium, provided the original work is properly cited.

\begin{abstract}
Background: Infertility affects about $10-15 \%$ of couples of reproductive age groups. The current evidence indicates a $9 \%$ prevalence of infertility with $56 \%$ of couples seeking medical care. Hysterolaparoscopy provides a comprehensive investigative procedure in which various factors causing female infertility can be assessed at one sitting.

Methods: A total 100 infertile women between 20-40 years of age including primary and secondary infertility were evaluated. Patients would be investigated thoroughly for infertility and in preparation for anaesthesia. Tests include follicular study, Ultrasound pelvis. Hysterolaparoscopy was performed in the pre ovulatory period between days 6-10 of the cycle for infertility evaluation.

Results: In the present study out of 100 cases for infertility evaluated, primary infertility were $57(57 \%)$ and secondary infertility were 43(43\%). In our study out of 100 patients, Hysterolaparoscopy showed tuberculosis in 24 $(24 \%)$ patients, remaining were endometriosis, polycystic ovarian syndrome, congestion, intra-pelvic adhesions, hydro-salpinx.

Conclusions: It is concluded that while treating the causes of female infertility combined simultaneous diagnostic laparoscopy and hysteroscopy should be performed in all infertile patients as" seeing is believing" and if any pathologies found to be operable the gynaecologist can perform operative hystero-laparoscopy at that time, hence anticipating the pathologies after pre-operative work up is very important.
\end{abstract}

Keywords: Follicular study, Hysteroscopy, Infertility, Laparoscopy

\section{INTRODUCTION}

Infertility is defined as failure to conceive during one year of unprotected frequent intercourse. It affects approximately $10-15 \%$ of couples. Leading cause of infertility includes tuboperitoneal disease (40-50\%), ovulatory disorders $(30-40 \%)$, uterine factor $(15-20 \%)$, male factor $30-40 \%$, and unexplained.

Hysterolaparoscopy is an effective and safe tool in comprehensive evaluation of infertility for diagnosis and further treatment of the lesions involving the pelvic structures. Treatment modalities at the time of laparoscopy comprised of adhesiolysis, ablation of endometriosis, ovarian drilling, and ovarian cystectomy. Hysteroscopic interventions were proximal tubal cannulation, septoplasty, synecolysis and myomectomy. ${ }^{1}$

Diagnostic hysterolaparoscopy is an effective diagnostic and therapeutic modality for certain significant and correctable abnormalities in pelvis, tubes and uterus which are missed by other imaging modalities. ${ }^{2}$ 
Diagnostic laparoscopy is the gold standard for diagnosing the tubal pathology, peritoneal factors, ovarian factors and uterine factors as cause of infertility Laparoscopic abnormalities are more common than hysteroscopic abnormalities both in primary infertility group and secondary infertility group. ${ }^{3}$ The prevalence of primary infertility was higher among women aged 20-24 years than among older women. ${ }^{4}$ Injuries can occur during the insertion of various instruments through the abdominal wall or during operative treatment. Conditions may increase the risk of serious complications; include previous abdominal surgery, adhesions, pelvic infections, obesity, or excessive thinness. Hysterolaparoscopy is the better choice for diagnosing the causative factor as well as treating the possible aetiologies.

\section{METHODS}

Women between 20-40 years of age, presenting with infertility from May-2016 to June 2018 were included in the study. The study was conducted after obtaining ethical clearance and after fulfilling the exclusion and inclusion criteria. Informed written consent was obtained from them. A detailed history was taken, and a thorough clinical examination was done.

This study was performed in a tertiary care teaching hospital, where well-established peri-operative prophylactic measures are rigorously followed. Detailed history would be taken from all patients complaining of infertility. Patient's husband's history in relation to occupation, significant medical or surgical history, any addictions, and coital history would be elicited.

Patients would be investigated thoroughly for infertility and in preparation for anaesthesia. Tests include complete blood count, liver function test, Renal function test; serum FSH, LH, PROLACTIN, TSH, follicular study, Ultrasound pelvis. Hysterolaparoscopy was performed in the pre ovulatory period between days 6-10 of the cycle for infertility evaluation. Pre-anaesthetic check up to be done prior to the procedure

On Hysteroscopy, uterine cavity would be examined for the presence of septum, any congenital malformation, fibrotic bands, polyps, myomas, endometrial appearance, thickness and color. Endocervical canal was visualized for any growth or polyps. Both the tubal ostia were visualized.

On Laparoscopy, pelvic cavity and organs were inspected. Uterus was inspected for its shape, size, position and surface. Cul-de-sac was examined for any adhesions, obliteration, endometriotic nodules or fluid. Ovaries were viewed for size, shape, surface, color, presence of cysts and relation with tubes. Fallopian tubes were inspected carefully for size, shape, surface, kinking, dilatation, stricture or hydrosalpinx. Laparoscopic chromopertubation was performed for testing tubal patency in which methylene blue dye will be injected with a $20 \mathrm{ml}$ syringe via Leech Wilkinson cannula and spillage of dye from the fimbrial end of tube visualized. The data of all the patients were statistically analysed at the end of the study, with respect to various parameters as detailed in the proforma.

\section{Inclusion criteria}

- All cases including primary and secondary infertility and who will give consent for surgery and who agree to participate in the study.

\section{Exclusion criteria}

- Infertile women with less than 20 years or more 40 years of age

- Patient who has associated male factor

- Patients who refuse surgery.

\section{RESULTS}

Out of 100 infertile patients $19(19 \%)$ patients were aged between 20-24 years, 42 (42\%) patients were aged were 25-29 years, 29 (29\%) patients were 30-34 years, 10 $(10 \%)$ patients were more than 35 years (Table 1 ).

Out of total 100 cases for infertility evaluated, primary infertility was $57(57 \%)$ and secondary infertility was 43 (43\%) (Table 2).

Table 1: Distribution of age among infertile patients.

\begin{tabular}{|lll|}
\hline Age $($ Years) & No. & Percentage \\
\hline 20 to 24 & 19 & $19.0 \%$ \\
\hline 25 to 29 & 42 & $42.0 \%$ \\
\hline 30 to 34 & 29 & $29.0 \%$ \\
\hline 35 and more & 10 & $10.0 \%$ \\
\hline Total & $\mathbf{1 0 0}$ & $\mathbf{1 0 0 . 0 \%}$ \\
\hline
\end{tabular}

Table 2: Distribution among the cases type of infertility.

\begin{tabular}{|lll|}
\hline Type of infertility & No. & Percentage \\
\hline Primary & 57 & $57.0 \%$ \\
\hline Secondary & 43 & $43.0 \%$ \\
\hline Total & $\mathbf{1 0 0}$ & $\mathbf{1 0 0 . 0 \%}$ \\
\hline
\end{tabular}

Table 3: Distribution among the cases of duration of infertility (years).

\begin{tabular}{|c|c|c|}
\hline Duration of infertility (Years) & No. & Percentage \\
\hline$<5$ & 34 & $34.0 \%$ \\
\hline 5 to 9 & 50 & $50.0 \%$ \\
\hline 10 to 14 & 13 & $13.0 \%$ \\
\hline 15 and more & 3 & $3.0 \%$ \\
\hline Total & 100 & $100.0 \%$ \\
\hline
\end{tabular}

Out of 100 patients $50(50 \%)$ patient infertile since 5-9 years, $13(13 \%)$ patients since $10-14$ years, $34(34 \%)$ 
patient less than 5 years, $3(3 \%)$ patients was infertile since 15 or more than 15 years (Table 3 ).

Table 4: Distribution among the cases of menstrual history.

\begin{tabular}{|l|l|l|}
\hline Menstrual history & No. & Percentage \\
\hline Irregular & 32 & $32.0 \%$ \\
\hline Regular & 68 & $68.0 \%$ \\
\hline Total & $\mathbf{1 0 0}$ & $\mathbf{1 0 0 . 0 \%}$ \\
\hline
\end{tabular}

Out of 100 infertile patients $68(68 \%)$ patients had regular menstrual cycle, $32(32 \%)$ patients had irregular cycles (Table 4).

Table 5: Distribution among the cases of significant obstetric history.

\begin{tabular}{|lll|}
\hline Significant obstetric history & No. & Percentage \\
\hline Previous curettage & 22 & $22.0 \%$ \\
\hline Previous live birth & 7 & $7.0 \%$ \\
\hline Medical abortion & 6 & $6.0 \%$ \\
\hline $\begin{array}{l}\text { Previous curratage, IUD, medical } \\
\text { abortion }\end{array}$ & 2 & $2.0 \%$ \\
\hline $\begin{array}{l}\text { Previous curratage, medical } \\
\text { abortion }\end{array}$ & 2 & $2.0 \%$ \\
\hline Ectopic pregnancy & 1 & $1.0 \%$ \\
\hline IUD & 1 & $1.0 \%$ \\
\hline $\begin{array}{l}\text { Previous curettage, ectopic } \\
\text { pregnancy }\end{array}$ & 1 & $1.0 \%$ \\
\hline Previous curettage, IUD & 1 & $1.0 \%$ \\
\hline Nulli-gravida & 57 & $57.0 \%$ \\
\hline Total & $\mathbf{1 0 0}$ & $\mathbf{1 0 0 . 0 \%}$ \\
\hline
\end{tabular}

Table 6: Distribution among the cases of past history.

\begin{tabular}{|c|c|c|}
\hline Past history & No. & Percentage \\
\hline Hypothyroid & 8 & $8.0 \%$ \\
\hline Tuberculosis & 6 & $6.0 \%$ \\
\hline Asthma & 4 & $4.0 \%$ \\
\hline Hypertension & 3 & $3.0 \%$ \\
\hline Adnexal surgery & 2 & $2.0 \%$ \\
\hline Jaundice & 2 & $2.0 \%$ \\
\hline Abdominal trauma & 1 & $1.0 \%$ \\
\hline Anxiety disorder & 1 & $1.0 \%$ \\
\hline Bicornuate, unicolis & 1 & $1.0 \%$ \\
\hline Diabetes mellitus & 1 & $1.0 \%$ \\
\hline PCOS & 1 & $1.0 \%$ \\
\hline Septum resection & 1 & $1.0 \%$ \\
\hline $\mathrm{Tb}$ contact & 1 & $1.0 \%$ \\
\hline Nil & 68 & $68.0 \%$ \\
\hline Total & 100 & $100.0 \%$ \\
\hline
\end{tabular}

Out of 100 patients $28(28 \%)$ patients had previous history of curettage, 7 (7\%) patients had previous live birth, $6(6 \%)$ patients had previous medical abortion, one (1\%) patient had ectopic pregnancy, one $(1 \%)$ patient had intra uterine insertion and $57(57 \%)$ patients had no previous pregnancy events (Table 5).

Out of 100 infertile patients, $8(8 \%)$ patients had hypothyroidism, $6(6 \%)$ patients had tuberculosis, 4 (4\%) patients had asthma, $3(3 \%)$ patients had hypertension, 2 (2\%) patients had previous adnexal surgery, 2 (2\%) patients had jaundice, one $(1 \%)$ patient had previous history of abdominal trauma, one $(1 \%)$ patient had anxiety disorder, one (1\%) patient was diagnosed case of bicornuate unicolis, one $(1 \%)$ patient had diabetes mellitus, one $(1 \%)$ patient had poly cystic ovarian disease, one (1\%) patient had TB contact and $68(68 \%)$ patients had no previous past history (Table 6).

Table 7: Distribution among the cases of per speculum.

\begin{tabular}{|lll|}
\hline Perspeculum & No. & Percentage \\
\hline Vaginitis & 12 & $12.0 \%$ \\
\hline Cervical erosion & 3 & $3.0 \%$ \\
\hline Pin point cervix & 2 & $2.0 \%$ \\
\hline Polyp & 1 & $1.0 \%$ \\
\hline Short cervix & 1 & $1.0 \%$ \\
\hline Healthy & 81 & $81.0 \%$ \\
\hline Total & $\mathbf{1 0 0}$ & $\mathbf{1 0 0 . 0 \%}$ \\
\hline
\end{tabular}

Out of 100 patients, 81 patients $(81 \%)$ healthy on speculum examination, 12 patients $(12 \%)$ had vaginitis, 3 patients $(3 \%)$ had cervical erosion, 2 patients $(2 \%)$ had pin point cervix, 1 patient (1\%) had polyp on examination, 1 patient (1\%) had short cervix (Table 7).

Table 8: Distribution among the cases of per vaginum.

\begin{tabular}{|lll|}
\hline Per vaginum & No. & Percentage \\
\hline Bulky & 10 & $10.0 \%$ \\
\hline Retroverted & 4 & $4.0 \%$ \\
\hline Adnexal mass & 3 & $3.0 \%$ \\
\hline Restricted mobility & 2 & $2.0 \%$ \\
\hline Small size & 3 & $3.0 \%$ \\
\hline Broad fundus & 1 & $1.0 \%$ \\
\hline Minimal tenderness & 1 & $1.0 \%$ \\
\hline Vaginal wall cyst & 1 & $1.0 \%$ \\
\hline Normal & 75 & $75.0 \%$ \\
\hline Total & $\mathbf{1 0 0}$ & $\mathbf{1 0 0 . 0 \%}$ \\
\hline
\end{tabular}

Table 9: Distribution among the cases of hormones.

\begin{tabular}{|lll|}
\hline Hormones & No. & Percentage \\
\hline Hyperprolactinemia & 8 & $8.0 \%$ \\
\hline Lh>Fsh & 8 & $8.0 \%$ \\
\hline Normal & 84 & $84.0 \%$ \\
\hline Total & $\mathbf{1 0 0}$ & $\mathbf{1 0 0 . 0 \%}$ \\
\hline
\end{tabular}

Authors studied that out of 100 patients $10(10 \%)$ patient had bulky uterus on per vaginal examination, 4 (4\%) patients had retroverted uterus, $3(3 \%)$ patients had 
palpable adnexal mass, $1(1 \%)$ patient had broad fundus felt on per-vaginal examination, mobility restricted in 2 (2\%) patients, minimal tenderness felt in $1(1 \%)$ patient, vaginal cyst present in 1 patient. $75(75 \%)$ patients had normal uterus and free fornices (Table 8 ).

Out of 100 patients, $8(8 \%)$ patient hormonal reports showed hyperprolactinemia, $8(8 \%)$ patients' reports showed raised LH level than FSH others had normal hormonal profile (Table 9).

Table 10: Distribution among the cases of follicular study.

\begin{tabular}{|c|c|c|}
\hline Follicular study & No. & Percentage \\
\hline Multiple small follicle & 28 & $28.0 \%$ \\
\hline Signs of ovulation & 57 & $57.0 \%$ \\
\hline Ovarian cyst & 15 & $15.0 \%$ \\
\hline Total & 100 & $100.0 \%$ \\
\hline
\end{tabular}

Authors studied 100 patient follicular study; it showed 28 (28\%) patients had multiple small follicles, $15(15 \%)$ patients had ovarian cyst of varying size, and 57 (57\%) patients had signs of ovulation (Table 10).

Table 11: Distribution among the cases of hysteroscopy.

\begin{tabular}{|lll|}
\hline Hysteroscopy & No. & Percentage \\
\hline Polypoidal endometrium & 14 & $14.0 \%$ \\
\hline Scanty endometrium & 8 & $8.0 \%$ \\
\hline Endometrial polyp & 5 & $5.0 \%$ \\
\hline Moderate endometrium & 4 & $4.0 \%$ \\
\hline Os stenosed & 4 & $4.0 \%$ \\
\hline Tubular cavity & 4 & $4.0 \%$ \\
\hline Septate uterus & 4 & $4.0 \%$ \\
\hline $\begin{array}{l}\text { Scanty endometrium, fibrosed } \\
\text { cavity }\end{array}$ & 3 & $3.0 \%$ \\
\hline Calcified endometrium & 2 & $2.0 \%$ \\
\hline Fibrotic band & 2 & $2.0 \%$ \\
\hline Profuse endometrium & 1 & $1.0 \%$ \\
\hline White patches over fundus & 1 & $1.0 \%$ \\
\hline Normal & 48 & $48.0 \%$ \\
\hline Total & $\mathbf{1 0 0}$ & $\mathbf{1 0 0 . 0 \%}$ \\
\hline
\end{tabular}

Out of 100 patients, total 48 (48\%) had normal hysteroscopic findings, 14 (14\%) patients had polypoidal endometrium, $8(8 \%)$ patients had scanty endometrium, 5 (5\%) patients had endometrial polyp, $4(4 \%)$ patient had moderate amount of endometrium, 2 (2\%) patient had calcified endometrium, 2 (2\%) patients had fibrotic band, 1 patient had profuse endometrium, $1(1 \%)$ patient had white patches over fundus, not able to negotiate in $4(4 \%)$ patient because of stenosed os (Table 11).

In the present study, laparoscopy showed tuberculosis in $24(24 \%)$ patients, endometriosis in $14(14 \%)$ patients, polycystic ovarian syndrome in 14 (14\%) patients, congestion in $7(7 \%)$ patients, intra-pelvic adhesions in 8 patients, hydrosalpinx in $3(3 \%)$ patients, right sided hydrosalpinx in 1 patient, ovarian cyst in 3 patients, paraovarian cyst in 3 patients, broad fundus in $2(2 \%)$ patients, fibroid in $2(2 \%)$ patients, tubo-ovarian mass in $2(2 \%)$ patients, ovarian anomalies in $1(1 \%)$ patient, normal in $15(15 \%)$ patients (Table 12$)$.

Table 12: Distribution among the cases of laparoscopy.

\begin{tabular}{|lll|}
\hline Laparoscopy & No. & Percentage \\
\hline Tuberculosis & 24 & $24.0 \%$ \\
\hline Endometriosis & 15 & $15.0 \%$ \\
\hline Poly cystic ovarian syndrome & 14 & $14.0 \%$ \\
\hline Congestion & 7 & $7.0 \%$ \\
\hline Intra-pelvic adhesions & 8 & $8.0 \%$ \\
\hline Hydrosalpinx & 3 & $3.0 \%$ \\
\hline Ovarian cyst & 3 & $3.0 \%$ \\
\hline Paraovarian cyst & 3 & $3.0 \%$ \\
\hline Broad Fundus & 2 & $2.0 \%$ \\
\hline Fibroid & 2 & $2.0 \%$ \\
\hline Tubo-ovarian mass & 2 & $2.0 \%$ \\
\hline Ovarian anomaly & 1 & $1.0 \%$ \\
\hline Rt terminal hydrosalpinx & 1 & $1.0 \%$ \\
\hline Normal & 15 & $15.0 \%$ \\
\hline Total & $\mathbf{1 0 0}$ & $\mathbf{1 0 0 . 0 \%}$ \\
\hline
\end{tabular}

Table 13: Distribution among the cases of operative procedure.

\begin{tabular}{|lll|}
\hline Operative procedure & No. & Percentage \\
\hline Drilling & 14 & $14.0 \%$ \\
\hline Adhesiolysis & 10 & $10.0 \%$ \\
\hline Cyst puncture & 7 & $7.0 \%$ \\
\hline Cystectomy & 6 & $6.0 \%$ \\
\hline Fulguration & 6 & $6.0 \%$ \\
\hline Biopsy & 2 & $2.0 \%$ \\
\hline Cervical dilation & 1 & $1.0 \%$ \\
\hline Nil & 54 & $54.0 \%$ \\
\hline Total & $\mathbf{1 0 0}$ & $\mathbf{1 0 0 . 0 \%}$ \\
\hline
\end{tabular}

Table 14: Distribution among the cases of dye test.

\begin{tabular}{|lll|}
\hline Dye test & No. & Percentage \\
\hline Bilateral spill & 68 & $68.0 \%$ \\
\hline Bilateral block & 13 & $13.0 \%$ \\
\hline Bilateral delayed spill & 6 & $6.0 \%$ \\
\hline One side delay, one side spill & 5 & $5.0 \%$ \\
\hline Unilateral spill & 4 & $4.0 \%$ \\
\hline Unilateral delayed spill & 3 & $3.0 \%$ \\
\hline Extravasation & 1 & $1.0 \%$ \\
\hline Total & $\mathbf{1 0 0}$ & $\mathbf{1 0 0 . 0 \%}$ \\
\hline
\end{tabular}

During the procedure laparoscopy, out of 100 patients, 14 (14\%) patients undergone drilling of ovarian cystic lesions, $10(10 \%)$ patients undergone Adhesiolysis, 7 
(7\%) patients undergone cyst puncture, $6(6 \%)$ patients undergone fulguration, $2(2 \%)$ patients biopsy, one patient had undergone dilatation of stenosed cervical os and no procedure was performed in $54(54 \%)$ patients (Table 13).

Table 15: Distribution among the cases of final diagnosis.

\begin{tabular}{|lll|}
\hline Final diagnosis & No. & Percentage \\
\hline Tuberculosis & 29 & $29.0 \%$ \\
\hline Endometriosis & 15 & $15.0 \%$ \\
\hline Poly cystic ovarian syndrome & 14 & $14.0 \%$ \\
\hline Pelvic inflammatory disease & 10 & $10.0 \%$ \\
\hline Hydrosalpinx & 3 & $3.0 \%$ \\
\hline Ovarian cyst & 3 & $3.0 \%$ \\
\hline Paraovarian cyst & 3 & $3.0 \%$ \\
\hline Adnexal mass & 3 & $3.0 \%$ \\
\hline Fibroid & 2 & $2.0 \%$ \\
\hline Uterine anomaly & 2 & $2.0 \%$ \\
\hline Ovarian anomaly & 1 & $1.0 \%$ \\
\hline Polyp & 1 & $1.0 \%$ \\
\hline Tubal pathology & 1 & $1.0 \%$ \\
\hline Normal & 13 & $13.0 \%$ \\
\hline Total & $\mathbf{1 0 0}$ & $\mathbf{1 0 0 . 0 \%}$ \\
\hline
\end{tabular}

Authors performed 100 patients chromo-perturbation test, out of 100 patients, $68(68 \%)$ patients had bilateral spill, $6(6 \%)$ patients had bilateral delayed spill, $5(5 \%)$ patients had one side delayed spill, one side free spill, 4 (4\%) patients had unilateral spill, $3(3 \%)$ patients had unilateral delayed spill, one (1\%) patient had extravasation of dye (Table 14).

Table 16: Distribution among the cases of complication of procedure.

\begin{tabular}{|ll|l|}
\hline Complication of procedure & No. & Percentage \\
\hline Spotting PV & 2 & $2.0 \%$ \\
\hline Trochar site infection & 2 & $2.0 \%$ \\
\hline Urine retention & 2 & $2.0 \%$ \\
\hline Paralytic ileus & 1 & $1.0 \%$ \\
\hline Trochar site bleeding & 1 & $1.0 \%$ \\
\hline Urinary tract infection & 1 & $1.0 \%$ \\
\hline Vomiting & 1 & $1.0 \%$ \\
\hline Nil & 90 & $90.0 \%$ \\
\hline Total & $\mathbf{1 0 0}$ & $\mathbf{1 0 0 . 0 \%}$ \\
\hline
\end{tabular}

In this study, out 100 patients 29 (29\%) patients diagnosed as tuberculosis, 15 (15\%) patients diagnosed as endometriosis, 14 (14\%) patients diagnosed as polycystic ovarian disease, $10(10 \%)$ patients diagnosed as pelvic inflammatory disease, $3(3 \%)$ patients had hydrosalpinx, 3 (3\%) patients had ovarian cyst, 3 (3\%) patients had para-ovarian cyst, $3(3 \%)$ patients had adnexal mass, 2 (2\%) patients had fibroids, $2(2 \%)$ patients had uterine anomaly, one patient had ovarian anomaly, one (1\%) patient had polyp, one (1\%) patient had tubal pathology, $13(13 \%)$ patients showed normal study (Table 15).

Authors studied 100 patients hysterolaparoscopy study, out of which $2(2 \%)$ patients developed spotting PV after the procedure, 2 (2\%) patients developed trochors site infection, $2(2 \%)$ patients developed urine retention, 1 (1\%) patient developed paralytic ileus, one $(1 \%)$ patient developed vomiting, one $(1 \%)$ patient developed urinary tract infection, 90 (90\%) patients not developed any complications (Table 16).

In our study, out of 100 patients, $28(28 \%)$ patients were advised anti Koch's treatment, 22 (22\%) patients advised for planned relations, 15 (15\%) patients advised.

Table 17: Distribution among the cases of plan of management.

\begin{tabular}{|lll|}
\hline Plan of management & No. & Percentage \\
\hline AKT & 28 & $28.0 \%$ \\
\hline Planned relations & 22 & $22.0 \%$ \\
\hline IVF & 15 & $15.0 \%$ \\
\hline COH with IUI & 14 & $14.0 \%$ \\
\hline Broad spectrum antibiotics & 9 & $9.0 \%$ \\
\hline Hormonal therapy & 7 & $7.0 \%$ \\
\hline GNRH analogues & 4 & $4.0 \%$ \\
\hline Septum resection & 1 & $1.0 \%$ \\
\hline Total & $\mathbf{1 0 0}$ & $\mathbf{1 0 0 . 0 \%}$ \\
\hline
\end{tabular}

In-vitro fertilisation technique, 14 (14\%) patients advised controlled ovarian stimulation with intra uterine insemination, 9 patients advised broad spectrum antibiotics, 4 (4\%) patients advised hormonal therapy, 7 (7\%) patients advised GnRH analogues, one (1\%) patient advised septum resection (Table 17).

\section{DISCUSSION}

In this study, out of 100 infertile patients 19 (19\%) patients were aged between 20-24 years, 42 (42\%) patients were aged were 25-29 years, 29 (29\%) patients were $30-34$ years, $10(10 \%)$ patients were more than 35 years, $50(50 \%)$ patient infertile since 5-9 years, 13 (13\%) patients since 10-14 years, 34 (34\%) patient less than 5 years, $3(3 \%)$ patients was infertile since 15 or more than 15 years.

In Mali K et al, study majority of the patients belonged to the age group of 26-30 years and $2 \%$ of patients were between age group of 36-40 years. Majority of the patients presented with primary infertility of less than or equal to 5 years of duration as the couples are very anxious to conceive after marriage..$^{5}$

In the present study out of 100 cases for infertility evaluated, primary infertility were 57 (57\%) and secondary infertility were $43(43 \%)$, which was in comparison with study group of Borchia YG et al, where 
primary infertility were $35 \quad(70 \%)$ and secondary infertility were $15(30 \%)$ out of 50 patients studied. ${ }^{6}$ Raksha et al observed that in primary infertility following hysteroscopic findings were more common than in secondary infertility. ${ }^{2}$

In this study, out of 100 patients, $14(14 \%)$ patients undergone drilling of ovarian cystic lesions, $10(10 \%)$ patients undergone adhesiolysis, 7 (7\%) patients undergone cyst puncture, $6(6 \%)$ patients undergone fulguration, $2(2 \%)$ patients biopsy, one patient undergone dilatation of stenosed cervical os and no procedure was performed in $54(54 \%)$ patients.

In the procedure of hysterolaparoscopy, laparoscopy helps the direct visualisation of upper abdomen, pathology of, uterus, fallopian tubes, peritoneum, ovaries and chromo-pertubation through the cervix for visualisation of tubal patency.

In this study out of 100 patients, Laparoscopy showed tuberculosis in $24(24 \%)$ patients, endometriosis in 14 (14\%) patients, polycystic ovarian syndrome in $14(14 \%)$ patients, congestion in $7(7 \%)$ patients, intra-pelvic adhesions in 8 patients, hydro-salpinx in $3(3 \%)$ patients, right sided hydrosalpinx in 1 patient, ovarian cyst in 3 patients, para-ovarian cyst in 3 patients, broad fundus in 2 (2\%) patients, fibroid in $2(2 \%)$ patients, tubo-ovarian mass in $2(2 \%)$ patients, ovarian anomalies in $1(1 \%)$ patient, normal in $15(15 \%)$ patients.

In sharma et al, study, hysteroscopic abnormalities revealed myoma and polyp in $10(7.7 \%)$ and synechiae in $5(3.8 \%)$, which was similar to other studies result. ${ }^{2}$

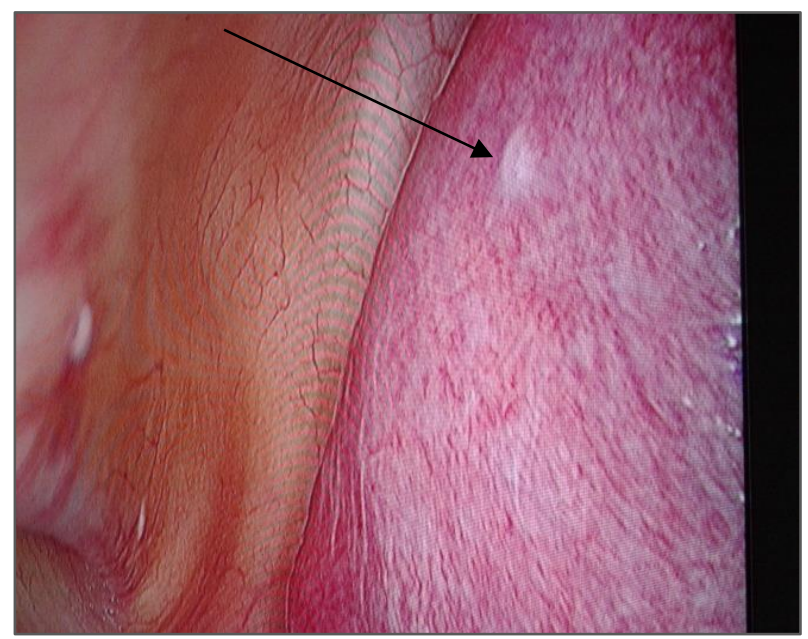

Figure 1: Tuberculosis, tubercle present over uterus.

Arrow points whitish elevated lesion, called tubercle present over the surface of the uterus. This is the important hallmark of tuberculosis.

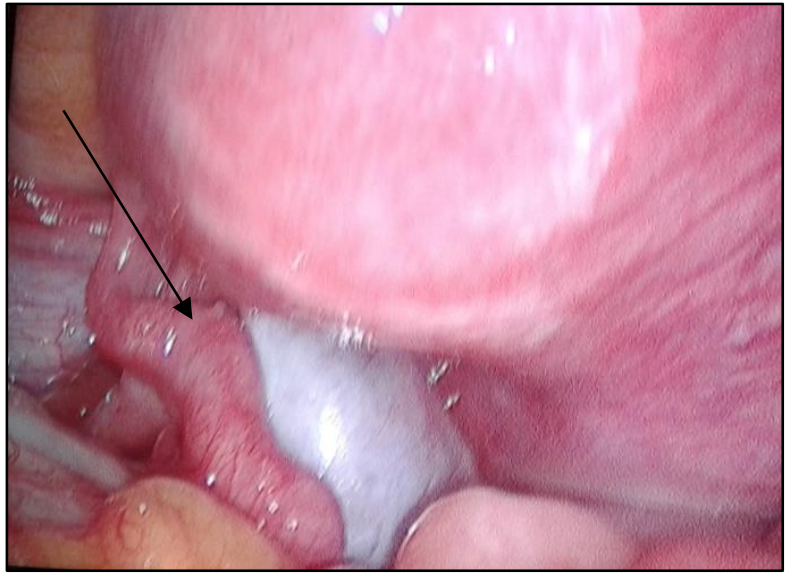

Figure 2: Tuberculosis of fallopian tube.

Arrow showed fallopian tube, which was affected by tuberculosis. Tube was congested, oedematous, tortuous, studded with tubercles.

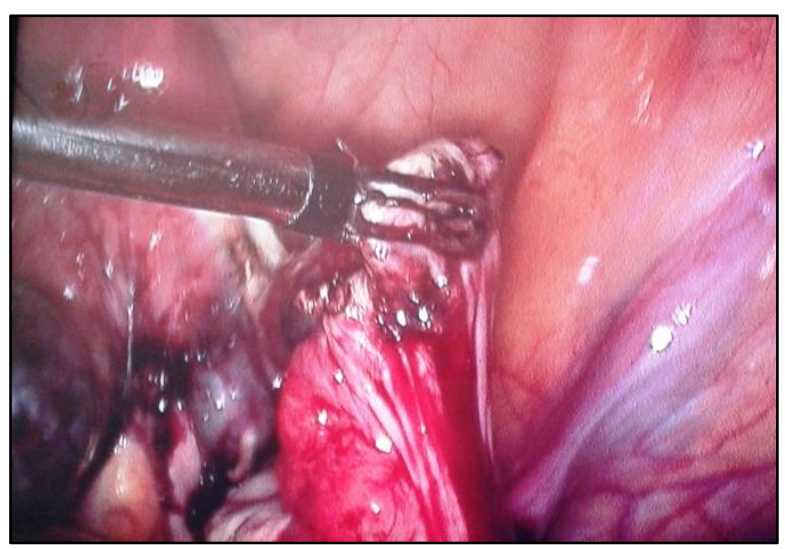

Figure 3: Endometriosis.

Laparoscopic grasper holds the adhesion present on the lateral wall of pelvic cavity. Adhesions were thick in consistency and presents with hemorrhagic spots.

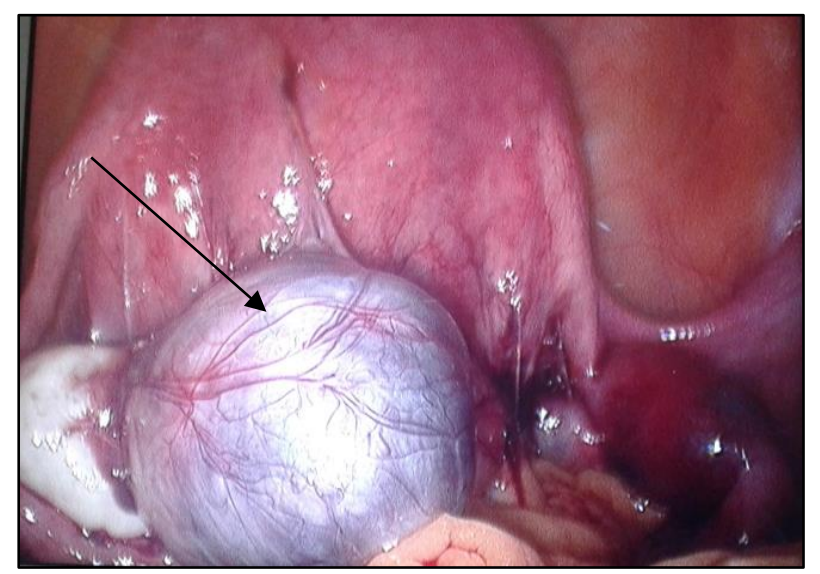

Figure 4: Simple ovarian cyst. 
Arrow showed the ovarian cyst with thin transparent wall and minimal vascularity present over the cyst surface. The cyst was punctured, drained and cyst wall was separated.

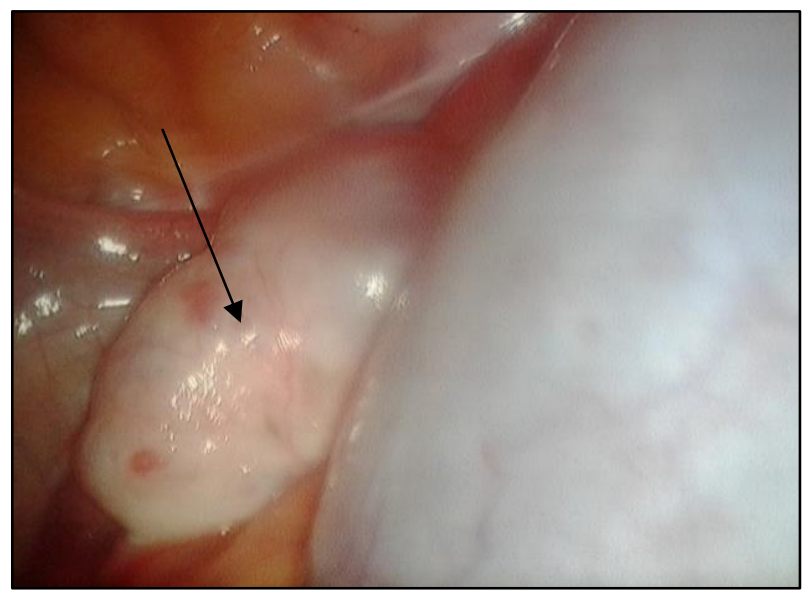

Figure 5: Poly cystic ovarian disease.

Laparoscopic view of ovarian pathology, here ovary was enlarged with multiple follicles. Later this poly cystic ovary was drilled and fluid drained.

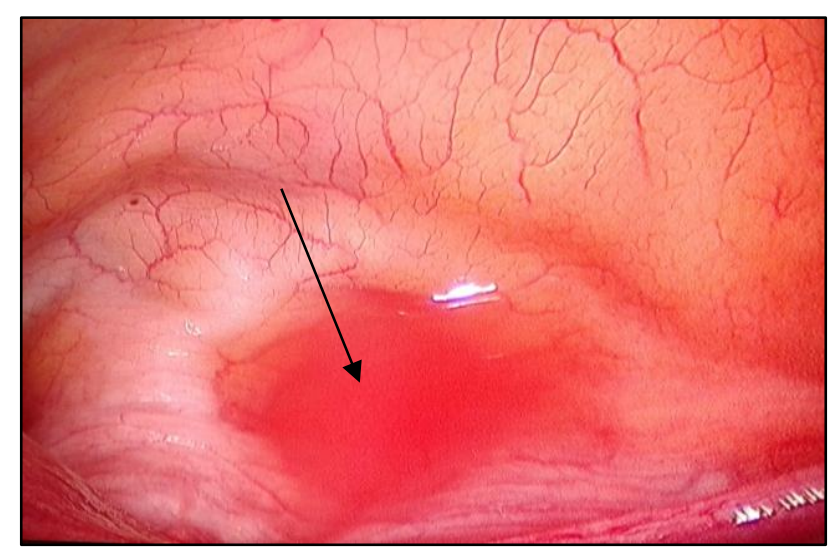

Figure 6: Pelvic inflammatory disease.

Arrow showed the straw colour fluid found on the pouch of Douglas, suggestive of pelvic inflammatory disease.

The results of Nayak et al study shows enlarged polycystic ovaries accounted for the most common laparoscopic abnormalities (9\%) followed by endometriosis $(7 \%)$ and tubal pathology $(6 \%)$. Myomas were seen in $4 \%$ cases, adenomyosis and adnexal pathology were detected in $3 \%$ of cases each. $20 \%$ of cases had uterine causes of infertility. ${ }^{7}$

Authors performed 100 patients chromo-perturbation test, out of 100 patients, $68(68 \%)$ patients had bilateral spill, $6(6 \%)$ patients had bilateral delayed spill, $5(5 \%)$ patients had one side delayed spill, one side free spill, 4 (4\%) patients had unilateral spill, 3 (3\%) patients had unilateral delayed spill, one (1\%) patient had extravasation of dye.

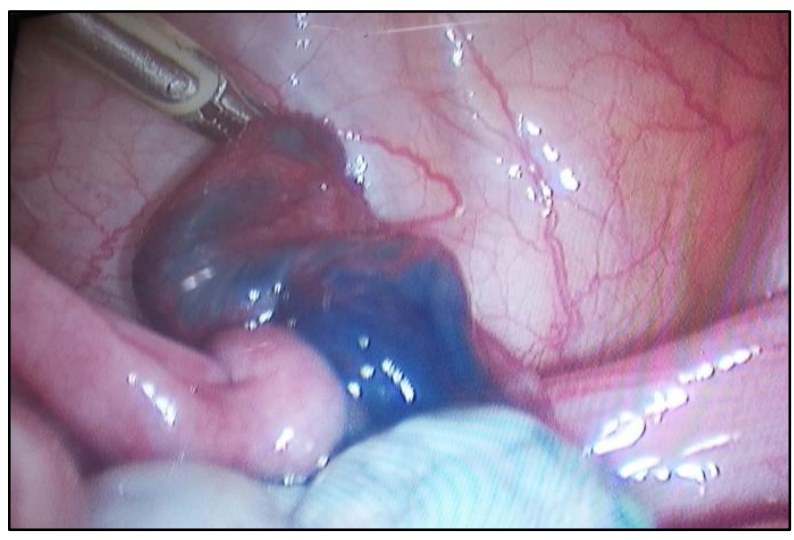

Figure 7: Chromopertubation-complete tubal block.

Laparoscopic picture showed the completely blocked tube with no spill of blue dye.

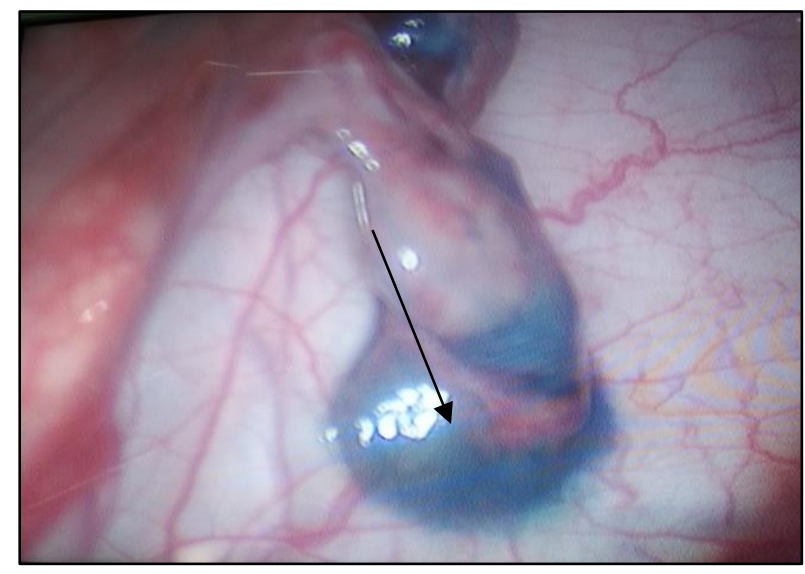

Figure 8: Chromopertubation- partial block.

Arrow showed the partial block, which is identified by delayed minimal spillage of tube.

Godinjak $\mathrm{Z}$ et al, conducted a retrospective study, they found bilateral tubes were blocked in $18(5 \%)$ and unilateral tubal occlusion were in $30(8,33 \%)$ of patients. Pelvic adhesions were revealed in $40(11.11 \%)$, and myomas in $42(11.65 \%)$ out of that $31(8.6 \%)$ were revealed by laparoscopy and $11(3.05 \%)$ by hysteroscopy. ${ }^{8}$ Singh R, et al, evaluated the role of diagnostic Hysterolaparoscopy in the management of infertile females.

The Laparoscopy was abnormal in $68 \%$ cases: Peritoneal and peri-tubal adhesions (29\%), phimosis/hydrosalpinx $(19 \%)$, polycystic ovaries $(26 \%)$, tubo-ovarian masses (16\%), endometriosis (4\%), congenital abnormalities $(10 \%)$, myomas (3\%), bilateral tubal blockage (49\%) and unilateral tubal blockage (17\%). ${ }^{9}$ Jayakrishnan et al, Prassant et al, from India detected findings of laparoscopy tubal blockages were present in $18 \%$ cases. 
7.29\% cases were having unilateral tubal blockage and $11.4 \%$ cases were having bilateral tubal block again. ${ }^{10}$ In Rai study, tubal occlusion was seen in $24 \%$ (unilateral) and $10 \%$ (bilateral). Rai et al found that the most common abnormality found on Laparoscopy was endometriosis (32\%). Among uterine factors, numbers of patients with fibroids were observed in $17(8.5 \%)$ patients followed by congenital anomalies in $6(3 \%)$ patients. ${ }^{11}$

In Dhanan jay study majority of the women with primary and secondary infertility did not had any complications ( $96.20 \%$ and $90.48 \%$ respectively).

Complication of hemorrhage $(2.53 \%)$ and infection $(1.27 \%)$ were noted in those with primary infertility while in women with secondary infertility haemorrhage was present in $4.76 \% .^{3}$ In general, studies have suggested that hysteroscopic removal of lesions $<2 \mathrm{~cm}$ does not adversely affect an IVF cycle. ${ }^{9}$ In Donnez study, total of 106 manuscripts were consulted. The incidence of myomas in infertile women without any obvious cause of infertility is estimated to be $1-2.4 \% .^{12}$

Authors studied 100 patients hysterolaparoscopy study, out of which 2 (2\%) patients developed spotting PV after the procedure, $2(2 \%)$ patients developed trochors site infection, 2 (2\%) patients developed urine retention, 1 (1\%) patient developed paralytic ileus, one $(1 \%)$ patient developed vomiting, one (1\%) patient developed urinary tract infection, $90(90 \%)$ patients not developed any complications.

In this study 90 (90\%) patients not developed any complications, only 10 patient's developed minor illness like spotting PV, trochors site infection, urine retention, paralytic ileus, vomiting, and urinary tract infection. Definitive procedure for reproductive tract pathology like, $14(14 \%)$ drilling of ovarian cystic lesions, $10(10 \%)$ Adhesiolysis, 7 (7\%) cyst puncture, $6(6 \%)$ patients undergone fulguration, $2(2 \%)$ biopsy, dilatation of stenosed cervical os and no procedure was performed in 54 (54\%) patients during Hysterolaparoscopy.

A combined modality of Hysterolaparoscopy helps in the management of infertility including primary and secondary infertility. With the help of Hysterolaparoscopy $28(28 \%)$ patients were advised anti Koch's treatment, 22 (22\%) patients adviced for planned relations, $15(15 \%)$ patients advised in-vitro fertilisation technique, $14(14 \%)$ patients advised controlled ovarian stimulation with intra uterine insemination, 9 patients advised broad spectrum antibiotics, $4(4 \%)$ patients advised hormonal therapy, 7 (7\%) patients advised GnRH analogues, one (1\%) patient advised septum resection.

Many diagnostic tests for female infertility have screening value but the gold standards are laparoscopy and simultaneous hysteroscopy. ${ }^{13}$

\section{CONCLUSION}

Hysterolaparoscopy is a gold standard in diagnosis and management of infertility. It is a feasible and acceptable procedure and it can be used as "One Time Approach" in the assessment of female infertility caused due to pelvic pathology.

It helps in diagnosing and management of certain factors causing infertility, which can be missed by any other method such as by USG, HSG and at same sitting it helps in operating the patients for pathologies hysteroscopicaly or laparoscopically like cystectomy, myomectomy, septum resection, pcos drilling etc.

It is concluded that while treating the causes of female infertility combined simultaneous diagnostic laparoscopy and hysteroscopy should be performed in all infertile patients as" seeing is believing" and if any pathologies found to be operable the gynaecologist can performs operative hystero-laparoscopy at that time, hence anticipating the pathologies after pre-operative work up is very important.

\section{Funding: No funding sources}

Conflict of interest: None declared

Ethical approval: The study was approved by the Institutional Ethics Committee

\section{REFERENCES}

1. Begum J, Samal S, Ghose S, Pallavee P, Samal R. Combined hysterolaparoscopy as an early option for initial evaluation of female infertility: a retrospective study of 135 patients. Int J Reprod Contracept Obstet Gynecol. 2015;4:584-8.

2. Raksha S, Gupta BD, Shah K. Role of diagnostic hysterolaparoscopy in evaluation of primary and secondary infertility. Int $\mathrm{J}$ Conte Med Res. 2016;3(3):768-72.

3. Shobha D, Madhu KN, Agrawal A. Role of diagnostic hysterolaparoscopy in evaluation of primary and secondary infertility. J Evolut Med Dent Sci. 2014;3(9):2194-207.

4. Mascarenhas MN, Flaxman SR, Boerma T, Vanderpoel S, Stevens GA. National, regional, and global trends in infertility prevalence since, 1990: a systematic analysis of 277 health surveys. PLoS Med. 2012;9(12):e1001356.

5. Mali K, Mohanty S. To study the role of hysteroscopy and laparoscopy in the evaluation of infertility. Int J Reprod Contracept Obstet Gynecol. 2016;5:3027-31.

6. Borchia YG, Sharma RK, Borchia BG, Mhapankar $\mathrm{S}$, Chaterjee A. Laparoscopy in 50 infertile couples prospective study. IJMCR. 2011:2(2):66.

7. Nayak PK, Mahapatra PC, Mallick JJ, Swain S, Mitra S, Sahoo J. Role of diagnostic hysterolaparoscopy in the evaluation of infertility: A 
retrospective study of 300 patients. J Hum Reprod Sci. 2013;6:32.

8. Godinjak Z, Idrizbegovic E. Should diagnostic hysteroscopy be a routine procedure during diagnostic laparoscopy in infertile women? JBMS. 2008;8:44-7.

9. Isikoglu M, Berkkanoglu M, Senturk Z, Coetzee K, Ozgur K. Endometrial polyps smaller than $1.5 \mathrm{~cm}$ do not affect ICSI outcome. Reprod Biomed Online. 2006;12(2):199-204.

10. Jayakrishnan K, Koshy AK, Raju R. Role of laparohysteroscopy in women with normal pelvic imaging and failed ovulation stimulation with intrauterine insemination. Hum Reprod Sc. 2010;3:20-4.

11. Rai A, Mishra MG. Diagnostic hystero-laparoscopy in work-up of female infertility. Int $\mathbf{J}$ Reprod Contracept Obstet Gynecol. 2017;6:2852-7.
12. Donnez J, Jadoul P. What are the implications of myomas on fertility? A need for a debate? Hum Reprod. 2002;17(6):1424-30.

13. Parveen S, Khanam M. Role of combined diagnostic laparoscopy and simultaneous diagnostic hysteroscopy for evaluation of female subfertility actors. J Surg Pak. 2010;15(1):44-7.

Cite this article as: Pandeeswari B., Valecha SM. A prospective study on the role of hysterolaparoscopy in the evaluation of infertility. Int $J$ Reprod Contracept Obstet Gynecol 2019;8:4766-74. 